\title{
Fabricating hydrophobic nanoparticles within mesoporous channel of silica for
}

\section{efficient TSNA removal}

Shuo Hao Li ${ }^{1,2}$, Xiao Dan Sun ${ }^{1}$, Lei-jun Wang ${ }^{2}$, Wen-bo Gu ${ }^{2}$, Wei-miao Wang ${ }^{2}$, Zheng Yu Yang ${ }^{2 *}$, Ying Wang ${ }^{1,3^{*}}$, Jian Hua Zhu 1* $^{*}$

${ }^{1}$ Key Laboratory of Mesoscopic Chemistry of MOE, College of Chemistry and Chemical Engineering, Nanjing University, Nanjing 210023, China, E-mail: jhzhu@ nju.edu.cn

2 Technology Center of Shanghai Tobacco (Group) Company, Shanghai 200082, China, E-mail: zyyang@sh.tobacco.com.cn

${ }^{3}$ Ecomaterials and Renewable Energy Research Center, Nanjing University, Nanjing 210093, China, E-mail: wangy@nju.edu.cn

Abstract Based on the synthesis of super hydrophobic silicic xerogel (SG) from methyltrimethoxysilane (MTMS) and dimethyldimethoxysilane (DMDMS), siliceous hydrophobic nanoparticles were fabricated within the mesoporous channels of SBA-15, in order to enhance the efficiency of adsorbing tobacco specific $N$-nitrosamines (TSNA) in aqueous solution. The concentration of precursor solution was adjusted to master the distribution of hydrophobic particles and the mean hydrophobicity of resulting composite, $150 \%$ increasing the capacity of SBA-15 in liquid adsorption of TSNA.

Keywords hydrophobic nanoparticles; mesoporous channels of silica; TSNA; liquid adsorption; environment protection

\section{Introduction}

Tobacco specific nitrosamines (TSNA) exist only in tobacco products, and they cause the potential 
risk of genetic mutations to induce tumor for smokers and passive smokers [1-4]. Most of the TSNA in cigarette smoke are transferred from tobacco $[5,6]$, so that reducing the content of TSNA in tobacco is effective to lower the content of the carcinogens in cigarette smoke. At present, one practical method of removing TSNA in tobacco products is to deal with tobacco by water extraction [7]. Therefore, it is possible to use liquid adsorption capturing the harmful substances like TSNA in tobacco extract solution, and the effective sorbent is the key to reduce the harm of tobacco on public health and environment.

In addition to common active carbon, zeolite can be used for liquid adsorption of TSNA [8-10]. However, the microporous channels of zeolite are labored to capture bulky TSNA (0.5-0.9 nm), and what's worse, most of commercial zeolites like $\mathrm{NaY}$ or $\mathrm{NaA}$ are hydrophilic. Harmful substances such as nitrosamine are mainly oily molecules and their adsorption in tobacco extract solution is inevitably interfered by water [9]. Therefore, new efficient sorbents of trapping TSNA are required having appropriate porous structure and certain hydrophobic to suppress the competition of water. Nowadays, it is common to modify mesoporous silica through alkylation with specific hydrophobic organic silicon reagents. The surface modification of mesoporous materials can be divided into one-pot synthesis and post impregnation methods [11]. One-pot synthesis is simple and able to make organic modifiers distributed more uniformly, but the mesoporous structure of the host will be degraded or damaged more or less due to the interference of organic agent in synthesis [12-14]. In addition, some organic modifiers are inevitably buried inside the channel wall and failed to modify the surface of sorbent [14]. On the contrary, post impregnation is usually able to maintain the favorable structure of mesoporous silica [11], and to adjust the size and surface curvature of channel in case using the optimal organic modifier with required size and functional groups.

Micron-sized super hydrophobic silicic xerogel (SG) has been simply synthesized with 
methyltrimethoxysilane (MTMS) and dimethyldimethoxysilane (DMDMS) [15], and the SG was modified by epitaxial growth of mesoporous silica to prepare a new capturer of $N$-nitrosamines in tobacco solution [16]. However, SG has a very small specific surface area $\left(4 \mathrm{~m}^{2} \mathrm{~g}^{-1}\right)$ though it owns an outstanding liquid adsorption performance in aqueous solution. Therefore, it will be valuable to divide the micron-sized SG into numerous nanoparticles and fabricate them inside the mesoporous channel in order to increase their efficiency of interception and adsorption TSNA in tobacco extract solution. To explore the influence of limited space on the fabrication and liquid adsorption of hydrophobic SG nanoparticles, two hosts are chosen including SBA-15 and MCM-41 mesoporous silica that represent the matrix with wide or narrow mesoporous channel. On the other hand, the silicon source concentration and composition of precursor solution are carefully adjusted to control the distribution of hydrophobic particles in the solid matrix with different geometric confined effects. Liquid adsorption of TSNA in tobacco extract solution is used here to assess the performance of these functional composites $[8,10]$, exploring the fabrication within mesoporous space.

\section{Experimental section}

\subsection{Materials}

Cetyltrimethylammonium bromide (CTAB) was the product of Nanjing Chemical (China). Tetraethylorthosilicate (TEOS) and P123 $\left(\mathrm{EO}_{20} \mathrm{PO}_{70} \mathrm{EO}_{20}\right)$ were purchased from Shanghai Wu-lian (China) and Aldrich, respectively. Burley and Virginia type tobacco fibers were bought in market, and other reagents were with AR grade and used as received.

SBA-15 was synthesized according to the recipes of literature [17]. In a typical synthesis, $3 \mathrm{~g}$ P123, $90 \mathrm{~g} \mathrm{HCl}(2 \mathrm{M})$ and $22.5 \mathrm{~g} \mathrm{H}_{2} \mathrm{O}$ were mixed at $298 \mathrm{~K}$. After P123 was dissolved, $6.38 \mathrm{~g}$ TEOS was added into the mixture at $313 \mathrm{~K}$, followed by vigorous stirring for $24 \mathrm{~h}$, and then this 
solution was transferred into a teflon bottle and heated statically at $373 \mathrm{~K}$ for another $24 \mathrm{~h}$. The solid product was recovered by filtration, washed with distilled water, air-dried and finally calcined at $773 \mathrm{~K}$ for $5 \mathrm{~h}$ to obtain SBA-15.

MCM-41 was also prepared according to literature $[9,18] .3 \mathrm{~g}$ silica aerosol and $1.5 \mathrm{~g} \mathrm{NaOH}$ were added to $45 \mathrm{~g}$ distilled water under stirring and defined as solution A, and solution B was prepared with $4.5 \mathrm{~g} \mathrm{CTAB}$ and $25 \mathrm{~g}$ distilled water. The solution B was added dropwise into solution $\mathrm{A}$ at $298 \mathrm{~K}$, and the $\mathrm{pH}$ value of mixture was adjusted to 9-11 using $\mathrm{HCl}$ solution (2 M). After stirring continuously for an additional $6 \mathrm{~h}$, the gel mixture was heated statically at $403 \mathrm{~K}$ for $72 \mathrm{~h}$. The solid was recovered by filtration, washed with distilled water, and air dried. Finally it was calcined at $823 \mathrm{~K}$ for $5 \mathrm{~h}$ to remove template.

\subsection{Synthesis of composited hydrophobic nanoparticles with mixed silanes}

$0.45 \mathrm{~g} \mathrm{CTAB}$ and $2.5 \mathrm{~g}$ urea were dissolved into certain amount of hydrochloric acid $(\mathrm{pH}=3.5)$ and stirred at $303 \mathrm{~K}$ for $0.5 \mathrm{~h}$. Then, $1.43 \mathrm{~g}$ MTMS and $0.84 \mathrm{~g}$ DMDMS were added in the solution and stirred for $0.5 \mathrm{~h}$, forming the precursor solution of super-hydrophobic silicic xerogel (SG). SBA-15 or MCM-41 matrix was put into the solution for impregnation, and after separation, the wet solid and the residual solution were heated at $353 \mathrm{~K}$ for $24 \mathrm{~h}$ separately. The solid was finally recovered by washed with distilled water, air-dried, giving the sample denoted as SS-r or SM-r, indicating the SG coated in SBA-15 or MCM-41, where $r$ was the volume ratio of hydrochloric acid to two silanes (MTMS+DMDMS) used in the synthesis (Table 1). And their weight increment was carefully measured (Table 2). The residual solution was also heated at $353 \mathrm{~K}$ for $24 \mathrm{~h}$ to give the solid sample named as $\mathrm{SG}^{\prime}[16]$.

Modification of mesoporous silica with single silane was performed in similar process, but the mixed precursor of MTMS and DMDMS was replaced by $2.39 \mathrm{~g}$ MTMS or $2.18 \mathrm{~g}$ DMDMS. The 
obtained sample was denoted as Sd-n, Sm-n, Md-n or Mm-n, indicating DMDMS or MTMS coated in SBA-15 or MCM-41, respectively, where $n$ was the volume ratio of hydrochloric acid to DMDMS or MTMS.

\subsection{Characterization}

X-ray diffraction (XRD) pattern of sample was recorded on an XRD-6000 diffractometer (power $40 \mathrm{kV}, 40 \mathrm{~mA}$ ) using $\mathrm{Cu}-\mathrm{Ka}$ radiation in the $2 \theta$ range of $0.6-10^{\circ}$. Nitrogen adsorption-desorption isotherm was obtained on a Micromeritics ASAP 2030 volumetric adsorption analyzer at $77 \mathrm{~K}$, for which $100 \mathrm{mg}$ of sample was evacuated at $363 \mathrm{~K}$ for $3 \mathrm{~h}$ in the degas port prior to test. Specific surface area of sample was calculated by the BET method using adsorption data acquired in the relative pressure range $\left(\mathrm{P} / \mathrm{P}_{0}\right)$ from 0.04 to 0.2 , while the total pore volume was determined from the amount adsorbed at a $\mathrm{P} / \mathrm{P}_{0}$ of about 0.99 . The morphology of sample was observed by SEM using a Hitachi S4800 FE-SEM system with $10 \mathrm{kV}$ accelerating voltage and 10 $\mathrm{mA}$ of beam current, and TEM analysis was carried out on a JEM-2100 electron microscope operating at $200 \mathrm{kV}$. Water contact angle was measured using the sessile drop method with a $3 \mu \mathrm{L}$ water droplet and a telescopic goniometer (Rame-Hart, Inc., MountainLake, NJ). FTIR spectrum was recorded in the range of 400-4000 $\mathrm{cm}^{-1}$ on a NEXUS870 spectrometer, for which the sample was mixed with $\mathrm{KBr}$ at a ratio of 3: $97(\mathrm{w} / \mathrm{w})$ and then pressed as a thin disk for testing. Thermal stability of samples was studied on STA-449F3 instrument working in argon and the temperature rose from $308 \mathrm{~K}$ to $1173 \mathrm{~K}$ at $10 \mathrm{~K} \mathrm{~min}^{-1}$.

\subsection{Liquid adsorption in tobacco extract solution}

Tobacco extract solution was prepared according to industrial processes: $500 \mathrm{~g}$ flue-cured tobacco stem (20\% long stem and $80 \%$ short stem) was submerged into $1150 \mathrm{~mL}$ water and the mixture was kept at $348 \mathrm{~K}$ for 30 min followed by filtration to separate fiber. On the other hand, 
$500 \mathrm{~g}$ tobacco fines (60\% burley tobacco and $40 \%$ flue-cured tobacco) were mixed with $3000 \mathrm{~mL}$ water at $293 \mathrm{~K}$ for $20 \mathrm{~min}$, and then filtrated to remove solid. Two solutions were mixed and enriched in rotary evaporator at $293 \mathrm{~K}$ with the rate of $90 \mathrm{r} \mathrm{min}^{-1}$ to reach the density of $1.2-1.22 \mathrm{~g}$ $\mathrm{cm}^{-3}$. The obtained tobacco extract solution was stored at $277 \mathrm{~K}$.

$50 \mathrm{mg}$ granular adsorbent was added into $10 \mathrm{~mL}$ tobacco extract solution in test tube and performed under dynamic and dark condition at $303 \mathrm{~K}$ for $1 \mathrm{~h}$. Thereafter it was centrifuged at 3000 rpm for 10 min to separate solid and solution $[8,16]$.

\subsection{Determination of TSNA in solution}

TSNA content of tobacco extract solution was determined by Applied Biosystems 4000 Q TRAP mass spectrum combined with an Agilent 1200 liquid chromatogram system equipped with a column of CAPCELL PAK C18 MG2 $(4.6 \mathrm{~mm} \times 150 \mathrm{~mm}, 3 \mu \mathrm{m})$. And the procedure and parameters used in the detection were same as that reported previously $[10,19]$.

\section{Results}

\subsection{Fabricating hydrophobic nanoparticles in mesoporous silica}

Fig. 1 presents the low-angle XRD patterns of SS-r and SM-r samples. SBA-15 sample showed three well-resolved XRD peaks indexed to the (100), (110) and (200) diffractions of the 2D hexagonal symmetry (p6mm) [17], but SS-4 sample only had one single peak of (100) diffraction with the slightly deviated position along with the obviously lowered intensity (Fig. 1a). As the amount of $\mathrm{HCl}$ solution used in the fabrication decreased, the characteristic peak further reduced on the pattern of SS-3 and almost disappeared on SS-2 sample. Clearly, the silicon precursor solution immersed and solidified inside the mesoporous channel of SBA-15 to form nanoparticles blocking the channel, leading to a strongly altered mesoporous structure similar to that modified with 3-aminopropyltriethoxysilane [20]. A different situation was found on MCM-41 sample that had 
three well-resolved XRD peaks indexed to the (100), (110) and (200) diffractions of hexagonal symmetry (Fig. 1b). Three coated derivers, SM-4, SM-3 and SM-2 all kept the single peak of (100) diffraction though the peak became weak as less acid was used in the fabrication. No doubt the ordered mesoporous structure of matrix is gradually damaged because of the declined diffraction intensity [21]. The siliceous modifiers inside channel inevitably reduced the scattering among wall of MCM-41, like the influence of carbon deposited in MCM-41 to cause irregular mesoporous structure [22].

Fig. 2 presents the nitrogen adsorption-desorption isotherms of SS-r composites. Parent SBA-15 sample had the typical IV isotherm with a large type H1 hysteresis loop and the narrow pore size distributions, mirroring a one-dimensional cylindrical straight channel [17]. However, the isotherm shape of three SS-r samples dramatically changed and hysteresis loop absent (Fig. 2a). Also, their surface areas sharply decreased from $793 \mathrm{~m}^{2} \mathrm{~g}^{-1}$ to less than $100 \mathrm{~m}^{2} \mathrm{~g}^{-1}$, and the thicker the concentration of precursor silane solution, the smaller the surface area. The pore volume of SS-r composites changed in the same tendency, too, and among them SS-2 sample exhibited the smallest $\left(25 \mathrm{~m}^{2} \mathrm{~g}^{-1}, 0.032 \mathrm{~cm}^{3} \mathrm{~g}^{-1}\right.$, Table 3) equaling to the $3 \sim 4 \%$ of that of parent SBA-15. The phenomenon meant the blocked or distorted channel of SBA-15 originating from the import of siliceous species, which is consistent with the results of XRD tests. Unlike the SBA-15 modified with (aminopropyl) triethoxysilane still kept IV isotherm and H1 hysteresis loop though the pore size decreased gradually [20], SS-r samples had the significantly damaged mesoporous structure, and one possible reason is the introduction of MTMS and DMDMS that not only interact with the surface hydroxyl groups of SBA-15, but trigger the polycondensation to form nanoparticles embedded inside channel. As a consequence, their surface area reduced drastically while the most probable pore size decreased from 6 to $3.2 \mathrm{~nm}$ (Fig. 2b). A strange phenomenon was observed on SM-r composites 
whose isotherms became freak (not shown) and surface area was no more than $10 \mathrm{~m}^{2} \mathrm{~g}^{-1}$, similar to that reported on the sample of MCM-48 modified with 3-propylchloridetriethoxysilane [9]. To explore whether the CTAB in precursor solution causes these changes, three SM-r samples were extracted with ethanol for $1 \mathrm{~h}$ and the resulting composites showed the normal isotherms of IV or I type (Fig. 2c and 2d), meanwhile their surface area rose to $150-655 \mathrm{~m}^{2} \mathrm{~g}^{-1}$ (Table 3). Besides, their pore sizes were close to that of parent MCM-41 $(3.5 \mathrm{~nm})$. Through these data, it is inferred that the CTAB micelles might locate inside channels and blocked the pore mouth, hindering the characterization of composite such as XRD test (Fig. S1). These micelles were flexible at ambient temperature but rigid at $77 \mathrm{~K}$ [23] so that the adsorption of nitrogen at $77 \mathrm{~K}$ was thus hindered. When SBA-15 was modified with single silane MTMS or DMDMS, its surface area decreased quickly from 793 to $6 \sim 26 \mathrm{~m}^{2} \mathrm{~g}^{-1}$ while the pore volume lowered from 0.796 to $0.02 \sim 0.05 \mathrm{~cm}^{3} \mathrm{~g}^{-1}$ (Table S1). Modification of MTMS made the surface area of MCM-41 declined from 1088 to around $20 \mathrm{~m}^{2} \mathrm{~g}^{-1}$ but DMDMS mage it further lowered to about $2 \mathrm{~m}^{2} \mathrm{~g}^{-1}$ (Table S1).

Fig. 3 displays the TEM images of SBA-15 and MCM-41 before and after hydrophobic modification. SBA-15 sample had a neat and uniform mesoporous structure in which the center distance of adjacent channel was about $6 \mathrm{~nm}$ [17]. Nonetheless, SS-3 sample showed a degraded structure whose regularity was considerably declined in which some channels were bended with rough surface, like the distorted channels of the SBA-15 in situ incorporated with titanium [24]. According to TEM image, the width of channels of SS-3 was significantly narrowed to about 2 3 $\mathrm{nm}$, and this decrease exceeded that observed on the SBA-15 loaded with bulky organosilicon reagents whose pore size lowered from 6.4 to $4.2 \mathrm{~nm}$ [14]. MTMS and DMDMS had small molecular groups like methyl or dimethyl groups, but they dramatically reduced the pore size of SS-3 sample through not only interaction with the silicon hydroxyl on channel wall, but also 
condensation polymerization of two silicon sources to form abundant nano-particles inside channel. In contrast, the channel width of SM-3 sample seemed similar to that of parent MCM-41 (Fig. 3e and $3 \mathrm{~g}$ ), though its regularity was slightly lowered accompanied with the channel wall became somewhat fuzzy and the vermiform structure emerged occasionally. Both SS-3 and SM-3 samples exhibited the neat six square channel orifices (Fig. 3f and 31), implying that they still remained symmetry plane of six-party $(\mathrm{p} 6 \mathrm{~mm})$ [20]. This phenomenon is different from that of Ti-modified SBA-15 whose channel orifices were obviously distorted [24], because the structure of SBA-15 is stable enough in this mild post-modification but easily changeable in one-pot synthesis. Another possible reason is the small molecular weight of two kinds of silicon reagents (MTMS and DMDMS). They easily insert and diffuse inside of the wild channel of SBA-15 so that their reactions occurred mainly in channel rather than in pore orifice. It is very hard to exactly measure the size of hydrophobic nanoparticles located inside the mesoporous channel. Yet, they could be introduced into the channels of SBA-15 (about $6 \mathrm{~nm})$ rather than that of MCM-41(3 nm) hence their theoretical size might be $3 \mathrm{~nm}$ or more. Also, these particles were guessed to have the size of around $3 \mathrm{~nm}$ on the basis of TEM image in Figure 3e.

Fig. 4 presents the FT-IR spectra of SBA-15, SS-3, MCM-41 and SM-3 samples. The bands at around 3434, 1637 and $961 \mathrm{~cm}^{-1}$ indicate the presence of -OH groups [25], which were significantly pronounced in SBA-15 and MCM-41 samples but slightly presented in SS-3 and SM-3 composites. It seems that MTMS and DMDM precursors had been consumed in polycondensation to fabricate hydrophobic nanoparticles in mesoporous silica. Furthermore, $\mathrm{C}-\mathrm{H}$ stretching vibration and bending bands appeared in the spectra of SS-3 and SM-3 samples at 2970 and $1270 \mathrm{~cm}^{-1}$, respectively, illustrating the methyl functional groups that lead to the hydrophobicity of samples [26]. Likewise, the band of Si-C bond at $848 \mathrm{~cm}^{-1}$ [27] only appeared in SS-3 and SM-3 samples. The strong peak 
near $1080 \mathrm{~cm}^{-1}$ and the weak peak near $800 \mathrm{~cm}^{-1}$ were assigned to the asymmetric and symmetric bending of Si-O-Si bonds, respectively [27]. SBA-15 and SS-3, or MCM-41 and SM-3, had different shape and intensity of peak, mirroring the mixed silanes had been consumed in the polycondensation to generate Si-O-Si bonds. Fig. 5 illustrates the TG-DSC curves of SBA-15, SS-3, MCM-41 and SM-3 samples. The weight loss under $400 \mathrm{~K}$, which presents the expulsion of water, of hydrophobic SS-3 and SM-3 samples were obviously smaller than that of SBA-15 and MCM-41, respectively. A sharp exothermic peak in DSC curve was observed at $782 \mathrm{~K}$ or $880 \mathrm{~K}$ for SS-3 or SM-3 sample, due to the oxidation of $\mathrm{Si}-\mathrm{CH}_{3}$ group to $\mathrm{Si}-\mathrm{OH}[25,27]$. Thus, it is inferred that the hydrophobic nanoparticles were fabricated in mesoporous silica.

Fig. 6 displays contact angle of SS-r and SM-r samples. Synchronically, the contact angle of all composites gradually decreased with the amount of acid rising, and the value of SS-4 was zero, which means the significant effect of silane solution's concentration on the hydrophobicity of resulting composites. Such influence also emerged on the coating amount (Table 2), especially in the test with glass sheets whose weight increment linearly decreased with the diluted silane solution (Table S2 and Fig. S2). Comparison of Table 2 with Table S2 reveals the effects of other factors on the coating of hydrophobic particles. The coating amount on SM-r was smaller than that of corresponding SS-r sample (Table 2), which ought to be ascribed to the limitation of narrow pore size because MCM-41 has a larger surface area than SBA-15 did (Table 3). Vertically, the hydrophobicity of SM-r samples were superior to that of corresponding SS-r (Fig. 6). The pore size of MCM-41 (3.3 nm) was smaller than that of SBA-15 (6 nm, Table 3) thus two silane agents were difficult to react inside the narrow channel. As the consequence, they were inclined to graft hydrophobic groups on MCM-41, resulting in the improved hydrophobic performance. When MCM-41 was modified with single silane MTMS or DMDMS, its contact angle rose to $120 \sim 129^{\circ}$ 
(Fig. S3) but the loading amount was lower than that of mixed silanes (Fig. S4). Single silane modification dramatically increased the contact angle of SBA-15 to $127 \sim 150^{\circ}$ (Fig. S3) though its loading amount (less than 40\%) obviously was inferior to that of mixed silanes (about 70\%, Fig. S4).

\subsection{Adsorption of TSNA in tobacco extract solution}

Fig. 7 presents the removal of TSNA in tobacco extract solution by two series of hydrophobic modified composites. On the whole, SBA-15 and MCM-41 exhibited the better liquid adsorption of TSNA after the hydrophobic modification. Among them SS-3 sample was the best because it could capture $25.9 \%$ of TSNA in tobacco extract solution, $151 \%$ higher than parent SBA-15 (10.3\%, Table 4), thanks to the enhanced hydrophobicity inhibiting competitive adsorption of water [9]. Besides, the narrowed channel of SS-3 sample, due to the insertion of hydrophobic nanoparticles, could provide a better geometric confined effect for these target carcinogens. MCM-41 itself showed a better adsorption than SBA-15 because of its inherent smaller pore size, but the improvement of hydrophobic modification became unobvious since SM-3 sample trapped only 6.7\% more TSNA than MCM-41 did (Table 4). In addition, the samples modified with single silane were assessed, but all of them had a low removal of TSNA under 10\%, even inferior to parent SBA-15 and MCM-41 samples (Table 4).

Different molecular configuration affects the adsorption of three components of TSNA, $N^{\prime}$-nitrosoanatabine (NAT), 4-(N-nitrosomethylamino)-1-(3-pyridyl)-1-butanone (NNK) and $N^{\prime}$-nitrosonornicotine (NNN), though they all have the functional $N$-nitroso group, N-NO [5]. NNK is a long strip-like molecule with the strength of $1.21 \mathrm{~nm}$ and the cross section of about $0.4 \mathrm{~nm}$ [10], but NAT and NNN are bulky ones with the cross section of about $0.8 \mathrm{~nm}$. All samples trapped more NAT than NNN or NNK due to the highest NAT content in the tobacco extract solution. As seen in 
Table 4, SS-3 sample had a more significant advantage in adsorbing NNN, namely the removal of NNN was enhanced from $1.8 \%$ (SBA-15, Table 4) to $20 \%$, though the capture of NNK and NAT were also obviously increased. Nonetheless, the reduction of NNK by SM-3 sample (9.6\%, Table 4) was lower than that by MCM-41 (15.8\%), which may related to the diluted silane solution used in the modification because a lower NNK reduction (7\%, Fig. 7) emerged on SM-4 sample that had been treated with the leaner silane solution (Table 1). MCM-41 lacked cation to attract the N-NO group of NNK hence this wirelike molecule could escape from the channel of sorbents. On the other hand, single hydrophobicity was not strong enough to trap NNK without assistance of geometric confinement, because the hydrophobic sample SG' only showed a weak ability in liquid adsorption of NNK (7.5\%, Table 4). Contrarily, when the volume ratio of acid to silanes achieved 3 in the preparation, almost modified composites displayed the best adsorption capacity of TSNA, which may relate to their optimal hydrophobicity consulting the results of contact angle (Fig. 6). Suitable hydrophobicity enables sorbent to suppress the competitive adsorption of water $[9,16]$, but makes the sorbent immersed in rather than floated on tobacco extract solution so that it can contact fully with TSNA.

\section{Discussion}

Compared with the SG modified with epitaxial grew mesoporous silica [16], the mesoporous silica coated with hydrophobic nanoparticles has some advantages for liquid adsorption of TSNA. The first is the large surface area thanks to the inherent characters of mesoporous silica. SBA-15 has a surface area over $700 \mathrm{~m}^{2} \mathrm{~g}^{-1}[9,19]$, affording a large space for coating hydrophobic nanoparticles. The second is the channel structure supplying the geometric confinement to limit thermal motion of target molecules $[9,10]$, and the third is the hydrophilicity that enables the sorbent immersed in aqueous solution to contact TSNA. The fourth is the mild conditions of 
impregnation under $353 \mathrm{~K}$ used here, in which the hydrophobic guest nanoparticles can be coated in mesoporous silica host to tailor both surface hydrophobicity and curvature of channel wall. Mesoporous silica SBA-15 did not have movable cation so that its adsorption depended on the weak interaction between TSNA and silicon hydroxyl [9,10]. Coating hydrophobic nanoparticles in mesoporous silica like SBA-15 is actually the multifunctional modification to conquer this drawback. These nanoparticles made the channel surface ups and downs, endowing smooth two-dimensional channel with the disordered multistage aperture (Scheme 1), which is beneficial for intercept of TSNA molecules [9] since the collision between target TSNA and adsorbent is increased. Moreover, the abundant methyl groups of hydrophobic nanoparticles inhibited competitive adsorption of water in aqueous solution and at the same time offered some hydrophobic sites to trap oily TSNA through the rule of similarity [16]. Consequently, SS-r samples exhibited an adsorption efficiency of TSNA significantly superior to SBA-15.

Silicon precursor solution is mixed with host matrix prior to formation of hydrophobic nanoparticles so that the size and distribution of the guests are enviably affected by the porous structure of host. Tiny spherical particles were faintly visible on the external surface of SM-3 but absent on the SEM image of SS-3 composite (Fig. S5). Together with the contact angle measured on the two samples (Fig. 6), it is inferred that the major nanoparticles were embedded inside the channel of SBA-15, but located on the external of MCM-41 probably due to its narrow channel. The limited nano-space may impede the polycondensation between MTMS and DMDMS, but further study is required to explore this impact.

Although SM-r had a similar adsorption capacity of TSNA to SS-r samples in tobacco extract solution (Fig. 6, Table 4), their advantage were different in comparison with their parent. The capacity of SS-3 sample (25.9\%) was $150 \%$ higher than that of SBA-15 (10.3\%) since the better 
geometric confined effect and proper hydrophobicity provided by the embedded hydrophobic nanoparticles. However, MCM-41 showed a better performance (16.5\%) than SBA-15 did because of its inherent smaller pore size to offer a tighter geometric constraints for the target $[9,10]$, hence the capacity of SM-3 sample (23.2\%) was $41 \%$ higher than that of MCM-41. Hydrophobic nanoparticles on the external surface of MCM-41 inhibited the competitive adsorption of water more or less, which is beneficial for TSNA to enter mesoporous channel and improve the liquid adsorption.

Those samples modified with single silane were just grafted some small molecular groups on the surface of host, and those groups like methyl and siloxane bridge ( $\mathrm{Si}-\mathrm{O}-\mathrm{Si}$ ) groups were reported to be 0.2 and $0.26 \mathrm{~nm}$ respectively [28]. Unlike SS-r samples with big hydrophobic nanoparticles occupied channel, these groups seem unable to provide an optimal geometric confinement and surface curvature for capture of TSNA carcinogens. In contrast, their high hydrophobicity $\left(120 \sim 150^{\circ}\right.$, Fig. S3) made them float on the surface of solution rather than immerse in it therefore all of them exhibited a relatively lower removal of TSNA than corresponding SS-3 or SM-3 sample, even inferior to SBA-15 and MCM-41 (Table 4). Proper hydrophobicity is important for liquid adsorption of TSNA in aqueous solution [9]. Hydrophobic adsorbent can reduce competitive adsorption of water, but it will float on solution failing to contact with TSNA [16]. Although the hydrophobicity of composite was gradually improved as the silanes precursor solution was concentrated (Fig. 6), the optimal adsorption of TSNA emerged on those prepared with the acid to silanes ratio of 3 (Fig. 7), which provides a valuable clue for functionization of mesoporous silica.

\section{Conclusion}

Hydrophobic nanoparticles were successfully fabricated in mesoporous silica SBA-15 to prepare 
TSNA trapper in tobacco extract solution, and the volume ratio of 3 of hydrochloric acid $(\mathrm{pH}=3.5$ ) to silanes was proven to be the optimal, which $150 \%$ increases the adsorption capacity of SBA-15 in tobacco extract solution.

Pore structure of host matrix affects the distribution of guest nanoparticles, and the majority of them located inside the channel of SBA-15 but on the external of MCM-41. The intrinsic structure of MCM-41 hinders the formation of hydrophobic nanoparticles inside its channel so that SBA-15 seemed to be the better host.

\section{Acknowledgment}

NSF of China (21173117, 21273106 and 21673113), Analysis Center of Nanjing University financially supported this research.

\section{Reference}

[1] S.S. Hecht, Mutat. Res: Fund. Mol. M., 1999, 424, 127-142.

[2] N.J. Farren, N. Ramirez, J.D. Lee, E. Finessi, A.C. Lewis, J.F. Hamilton, Environ. Sci. Technol. $2015,49,9648-9656$.

[3] Y-H. Cho, H-S. Shin, Anal. Methods, 2015, 7, 4472-4480

[4] C. Jansson, A. Paccon, B-G. Osterdahl, J. Chromatogr. A, 2003, 1008, 135-143.

[5] D. Hoffmann, M. Dong, S.S. Hecht, J. Natl, Cancer Inst.,1977, 58, 1841-1844.

[6] J.D. Adams, S.J. Lee, N. Vinchkoski, A. Castonguay, D. Hoffmann, Cancer Lett.,1983, 17, 339-346.

[7] S. Tatsuoka, Patent No. EP1782702 A1 (2005).

[8] X.D. Sun, W.G. Lin, L.J. Wang, B. Zhou, X.L. Lv, Y. Wang, S.J. Zheng, W.M. Wang, Y.G. Tong, J.H. Zhu, Microporous Mesoporous Mater. 2014, 200, 260-268.

[9] F. Wei, J.Y. Yang, L. Gao, F.N. Gu, J.H. Zhu, J. Hazard. Mater.2009, 172, 1482-1490. 
[10] W.G. Lin, B.C. Huang, B. Zhou, X.L. Lv, L.J. Wang, S.J. Zheng, W.M. Wang, Y.G. Tong, Y. Wang, J.H. Zhu, J. Porous Mater., 2014, 21, 311-320.

[11] T. Yokoi, H. Yoshitake, T. Tatsumi, J. Mater. Chem., 2004, 14, 951-957.

[12] T. Yokoi, H. Yoshitake, T. Tatsumi, Chem. Mater. 2003, 15, 4536-4538.

[13] O. Olkhovyk, M. Jaroniec, J. Am. Chem. Soc., 2005, 127, 60-61.

[14] M.M. Wan, L. Gao, Z. Chen, Y.K. Wang, Y. Wang, J.H. Zhu. Microporous Mesoporous Mater, $2012,155,24-33$

[15] G. Hayase, K. Kanamori, K. Nakanishi, J. Mater. Chem. 21 (2011),17077-17079.

[16] S.H. Li, W.G. Lin, B.C. Huang, J.H. Zhu, Microporous Mesoporous Mater. 2016, 227, 161-168.

[17] D.Y. Zhao, J.L. Feng, Q.S. Huo, N. Melosh, G.H. Fredrickson, B.F. Chmelka, G.D. Stucky, Science, 1998, 279, 548-552.

[18] P. Van Der Voort, P.I. Ravikovitch, K.P.D. Jong, M. Benjelloun, E.V. Bavel, A.H. Janssen, A.V.

Neimark, B.M. Weckhuysen, E. F. Vansant, J. Phys. Chem. B 2002, 106, 5873-5877.

[19] K.A. Wagner, N.H. Finkel, J.E. Fossett, I.G. Gillman, Anal. Chem., 2005, 77, 1001-1006.

[20] W.J. Qian, M.M. Wan, W.G. Lin, J.H. Zhu, J. Mater. Chem. B, 2014,2, 92-101.

[21] S. Van Donk, A.H. Janssen, J.H. Bitter, K.P. de Jong, Catal. Rev. 2003, 45, 297-319.

[22] J.Y. Yang, J. Yang, Y. Zhou, W.G. Lin, H.J. Wang, J.H. Zhu, J. Hazard. Mater. 2010, 176, 602-608.

[23] X.Y.M. Dong, Y.Y. Li, F. Wei, Y. Zhou, S.L. Zhou, J.H. Zhu, RSC Adv. 2015, 5, 5494-5500.

[24] M.M. Wan, X.D. Sun, W.J. Qian, S. Liu, J. Ma, J.H. Zhu, Microporous Mesoporous Mater. 2014, 199, 40-49.

[25] S. Cui, Y. Liu, M.H. Fan, Mater. Lett., 2011, 65, 606-609. 
[26] A.V. Rao, S.S. Latthe, D.Y. Nadargi, H. Hirashima, V. Ganesan, J. Colloid. and Interface Sci. 2009, 332, 484-490.

[27] S. Lee, Y.C. Cha, H.J. Hwang, Mater. Lett., 2007, 61, 3130-3133.

[28] A. Philip, K.R. Kumar, Bull. Mater. Sci., 2010, 33, 97-102. 
Table 1 The composition of precursor solution to coat hydrophobic particles.

\begin{tabular}{cccccccccc}
\hline & $\begin{array}{c}\text { CTAB } \\
(\mathrm{g})\end{array}$ & $\begin{array}{c}\text { Urea } \\
(\mathrm{g})\end{array}$ & $\begin{array}{c}\text { MTMS } \\
(\mathrm{g})\end{array}$ & $\begin{array}{c}\text { DMDMS } \\
(\mathrm{g})\end{array}$ & $\begin{array}{c}\mathrm{V}_{\text {MTMS }} \\
(\mathrm{mL})\end{array}$ & $\begin{array}{c}\mathrm{V}_{\text {DMDMS }} \\
(\mathrm{mL})\end{array}$ & $\begin{array}{c}\mathrm{V}_{\text {Mix }} \\
(\mathrm{mL})\end{array}$ & $\begin{array}{c}\mathrm{V}_{\mathrm{HCl}} \\
(\mathrm{mL})\end{array}$ & $\begin{array}{c}\mathrm{V}_{\text {HCl }}: \mathrm{V}_{\text {Mix }} \\
(\mathrm{n})\end{array}$ \\
\hline 1 & 0.46 & 2.5 & 1.43 & 0.84 & 0.965 & 1.497 & 2.5 & 5 & 2 \\
2 & 0.46 & 2.5 & 1.43 & 0.84 & 0.965 & 1.497 & 2.5 & 7.5 & 3 \\
3 & 0.46 & 2.5 & 1.43 & 0.84 & 0.965 & 1.497 & 2.5 & 10 & 4 \\
\hline
\end{tabular}

Table 2 The weight increase of samples after coated with hydrophobic particles.

\begin{tabular}{ccccc}
\hline Samples & $\mathrm{M}_{0}(\mathrm{~g})$ & $\mathrm{M}_{1}(\mathrm{~g})$ & $\triangle \mathrm{M}(\mathrm{g})$ & Loading $(\%)$ \\
\hline SS-2 & 0.1057 & 0.1782 & 0.0725 & 68.6 \\
SS-3 & 0.1048 & 0.1762 & 0.0714 & 68.1 \\
SS-4 & 0.1050 & 0.1719 & 0.0669 & 63.7 \\
SM-2 & 0.1053 & 0.1634 & 0.0581 & 55.2 \\
SM-3 & 0.1052 & 0.1506 & 0.0454 & 43.2 \\
SM-4 & 0.1055 & 0.1438 & 0.0383 & 36.3 \\
\hline
\end{tabular}

Table 3 The textural properties of mesoporous silica samples.

\begin{tabular}{cccccc}
\hline Sample & $\mathrm{S}_{\mathrm{BET}}{ }^{\mathrm{a}}\left(\mathrm{m}^{2} \mathrm{~g}^{-1}\right)$ & $\mathrm{S}_{\text {micro }}{ }^{\mathrm{b}}\left(\mathrm{m}^{2} \mathrm{~g}^{-1}\right)$ & $\mathrm{V}_{\mathrm{p}}^{\mathrm{c}}\left(\mathrm{cm}^{3} \mathrm{~g}^{-1}\right)$ & $\mathrm{V}_{\text {micro }}{ }^{\mathrm{d}}\left(\mathrm{cm}^{3} \mathrm{~g}^{-1}\right)$ & $\mathrm{D}_{\mathrm{p}}^{\mathrm{e}}(\mathrm{nm})$ \\
\hline SBA-15 & 793 & 138 & 0.796 & 0.054 & 6.0 \\
SS-2 & 25 & - & 0.032 & - & 3.2 \\
SS-3 & 51 & - & 0.068 & - & 3.2 \\
SS-4 & 78 & - & 0.139 & - & 3.4 \\
MCM-41 & 1088 & - & 0.672 & - & 3.3 \\
SM-2 & 2 & - & - & 0.004 & - \\
SM-3 & 6 & - & - & 0.003 & - \\
SM-4 & 10 & 6.8 & - & 0.003 & - \\
SM-2* & 150 & 15 & 0.108 & 0.004 & 3.54 \\
SM-3* & 242 & - & 0.164 & - & 3.30 \\
SM-4* & 655 & - & 0.370 & - & 3.30 \\
\hline
\end{tabular}

[a] $\mathrm{S}_{\mathrm{BET}}$, BET surface area; [b] $\mathrm{S}_{\text {mic }}$, micropore area; [c] $\mathrm{V}_{\mathrm{p}}$, total pore volume; [d] $\mathrm{V}_{\text {mic }}$, micropore volume; [e] $\mathrm{D}_{\mathrm{p}}$, $\mathrm{BJH}$ mesopore diameter calculated from the adsorption branch.

* This sample was extracted with ethanol at room temperature for $1 \mathrm{~h}$. 
Table 4 Residual TSNA in the tobacco extract solution adsorbed by porous sorbents.

\begin{tabular}{|c|c|c|c|c|c|c|c|c|}
\hline Samples & $\begin{array}{c}\mathrm{NNN}^{\mathrm{a}} \\
\left(\mathrm{nmol} \mathrm{g}^{-1}\right)\end{array}$ & $\begin{array}{l}\text { Cut } \\
(\%)\end{array}$ & $\begin{array}{c}\mathrm{NAT}^{\mathrm{b}} \\
\left(\mathrm{nmol} \mathrm{g}^{-1}\right)\end{array}$ & $\begin{array}{l}\text { Cut } \\
(\%)\end{array}$ & $\begin{array}{c}\mathrm{NNK}^{\mathrm{c}} \\
\left(\mathrm{nmol} \mathrm{g}^{-1}\right)\end{array}$ & $\begin{array}{l}\text { Cut } \\
(\%)\end{array}$ & $\begin{array}{c}\text { TSNA } \\
\left(\mathrm{nmol} \mathrm{g}^{-1}\right)\end{array}$ & $\begin{array}{c}\text { Reduced } \\
(\%)\end{array}$ \\
\hline Control & 0.127 & - & 0.268 & - & 0.055 & - & 0.450 & - \\
\hline${ }^{\#}$ Control & 0.130 & - & 0.141 & - & 0.082 & - & 0.352 & - \\
\hline SBA-15 & 0.125 & 1.8 & 0.232 & 13.4 & 0.047 & 14.9 & 0.404 & 10.3 \\
\hline SS-3 & 0.102 & 20.0 & 0.189 & 29.4 & 0.042 & 22.8 & 0.333 & 25.9 \\
\hline MCM-41 & 0.112 & 12.0 & 0.218 & 18.8 & 0.046 & 15.8 & 0.376 & 16.5 \\
\hline SM-3 & 0.108 & 15.1 & 0.188 & 29.8 & 0.050 & 9.6 & 0.346 & 23.2 \\
\hline $\mathrm{SG}^{\prime}$ & 0.124 & 2.4 & 0.233 & 13.1 & 0.051 & 7.3 & 0.408 & 9.3 \\
\hline${ }^{\#} \mathrm{Sm}-2$ & 0.126 & 2.6 & 0.132 & 6.4 & 0.083 & 0 & 0.341 & 3.3 \\
\hline${ }^{\#} \mathrm{Sm}-3$ & 0.122 & 6.1 & 0.124 & 12.0 & 0.083 & 0 & 0.328 & 6.8 \\
\hline${ }^{\#} \mathrm{Sm}-4$ & 0.124 & 4.3 & 0.127 & 9.8 & 0.073 & 10.6 & 0.324 & 7.9 \\
\hline${ }^{\#}$ Sd-2 & 0.125 & 3.5 & 0.134 & 4.5 & 0.080 & 2.9 & 0.339 & 3.7 \\
\hline${ }^{\#} \mathrm{Sd}-3$ & 0.124 & 4.8 & 0.132 & 6.0 & 0.079 & 3.5 & 0.335 & 4.9 \\
\hline${ }^{\#} \mathrm{Sd}-4$ & 0.127 & 2.2 & 0.131 & 6.8 & 0.080 & 2.9 & 0.338 & 4.1 \\
\hline${ }^{\#} \mathrm{Mm}-2$ & 0.126 & 3.0 & 0.135 & 3.8 & 0.083 & 0 & 0.345 & 2.1 \\
\hline${ }^{\#} \mathrm{Mm}-3$ & 0.125 & 3.5 & 0.131 & 7.1 & 0.077 & 5.9 & 0.333 & 5.4 \\
\hline${ }^{\#} \mathrm{Mm}-4$ & 0.125 & 3.9 & 0.132 & 6.0 & 0.075 & 8.8 & 0.332 & 5.8 \\
\hline${ }^{\#} \mathrm{Md}-2$ & 0.131 & 0 & 0.135 & 3.8 & 0.078 & 4.7 & 0.345 & 2.0 \\
\hline${ }^{\#} \mathrm{Md}-3$ & 0.122 & 6.1 & 0.134 & 4.9 & 0.081 & 1.2 & 0.337 & 4.4 \\
\hline${ }^{\#} \mathrm{Md}-4$ & 0.122 & 6.1 & 0.128 & 9.0 & 0.076 & 7.1 & 0.326 & 7.4 \\
\hline
\end{tabular}

${ }^{\text {a }} \mathrm{NNN}$ is $N^{\prime}$-nitrosonornicotine.

${ }^{\mathrm{b}}$ NAT is $N$-nitrosoanatabine.

${ }^{\mathrm{c}} \mathrm{NNK}$ is 4-(N-nitrosomethylamino)-1-(3-pyridyl)-1-butanone.

${ }^{\#}$ Those samples are adsorbed the corresponding letter solution.

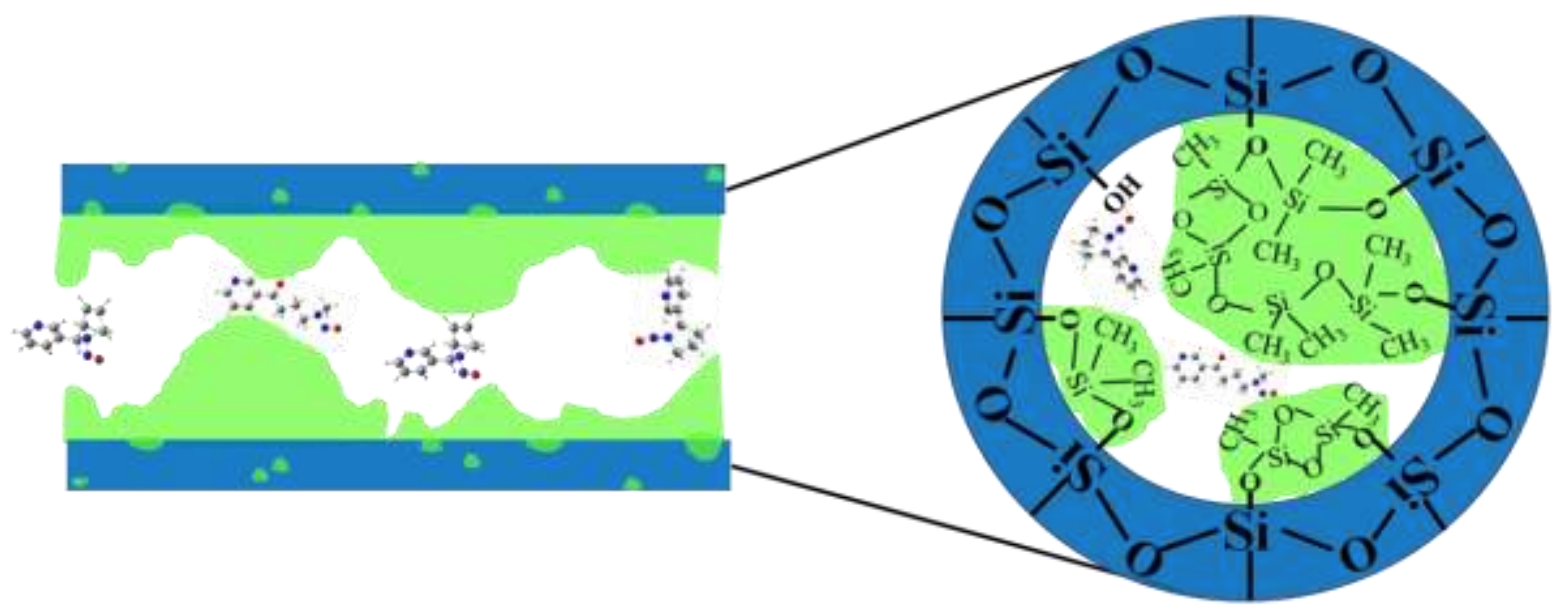

SBA-15

TSNA

Hydrophobic nanoparticles

Scheme 1 Hydrophobic nanoparticles within mesoporous channel of silica along with the adsorption of TSNA 


\section{Caption of Figures}

Fig. 1. Low-angle XRD patterns of (a) SBA-15 and (b) MCM-41 samples. .

Fig. 2.The $\mathrm{N}_{2}$ adsorption isotherm of (a, b) SBA-15 and (c, d) MCM-41 composites.

Fig. 3.TEM images of (a, b, c) SBA-15 and (d, e, f) SS-3 and (g, h, i) MCM-41 and (j, k, 1) SM-3 samples.

Fig. 4. FT-IR spectra of SBA-15, SS-3, MCM-41 and SM-3 samples.

Fig. 5. The TG-DSC plot of (a) SBA-15 and SS-3, (b) MCM-41 and SM-3 samples.

Fig. 6. Contact angle images of (a) SM-2, (b) SM-3, (c) SM-4, (d) SS-2, (e) SS-3 and (f) SS-4 samples.

Fig. 7. Adsorption of TSNA by SS-r (a) and SM-r samples in tobacco extract solution. 

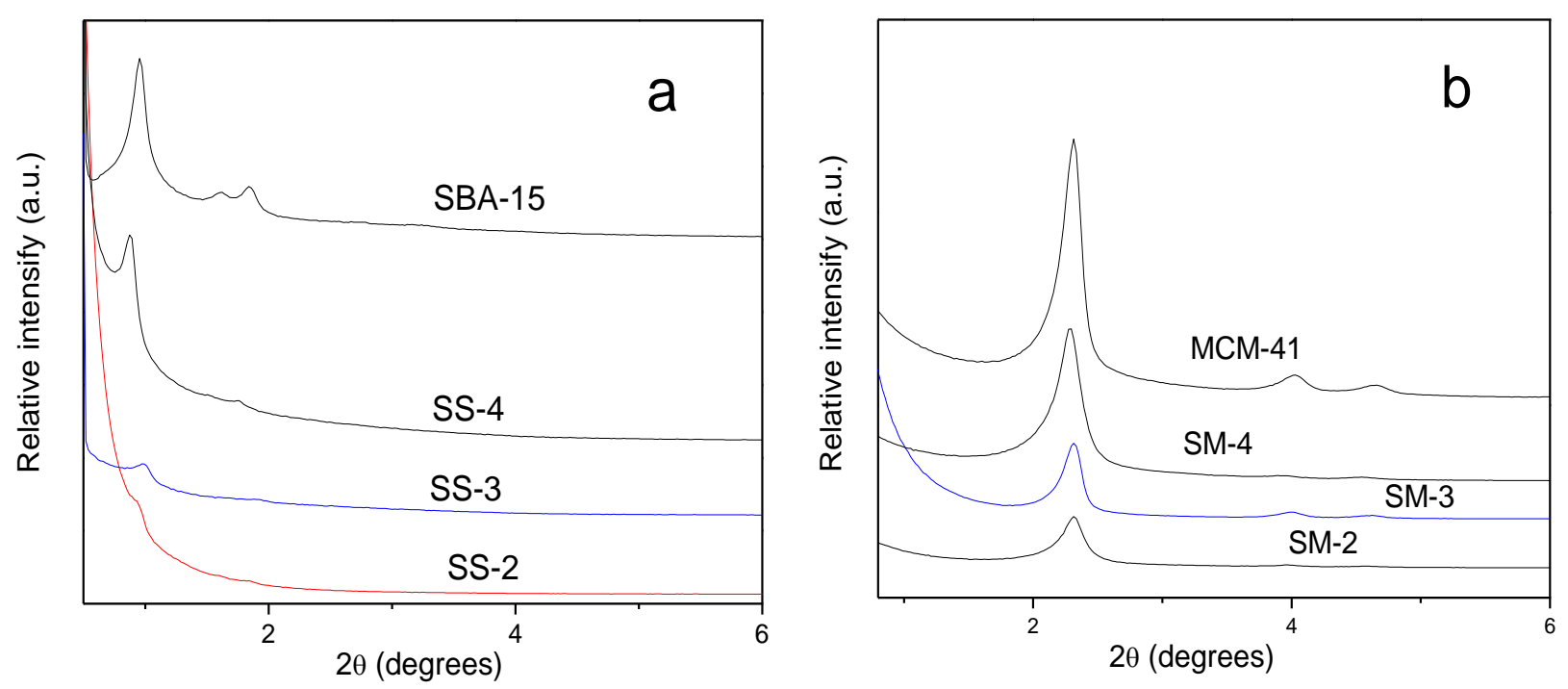

Fig. 1. Low-angle XRD patterns of (a) SBA-15 and (b) MCM-41 samples.
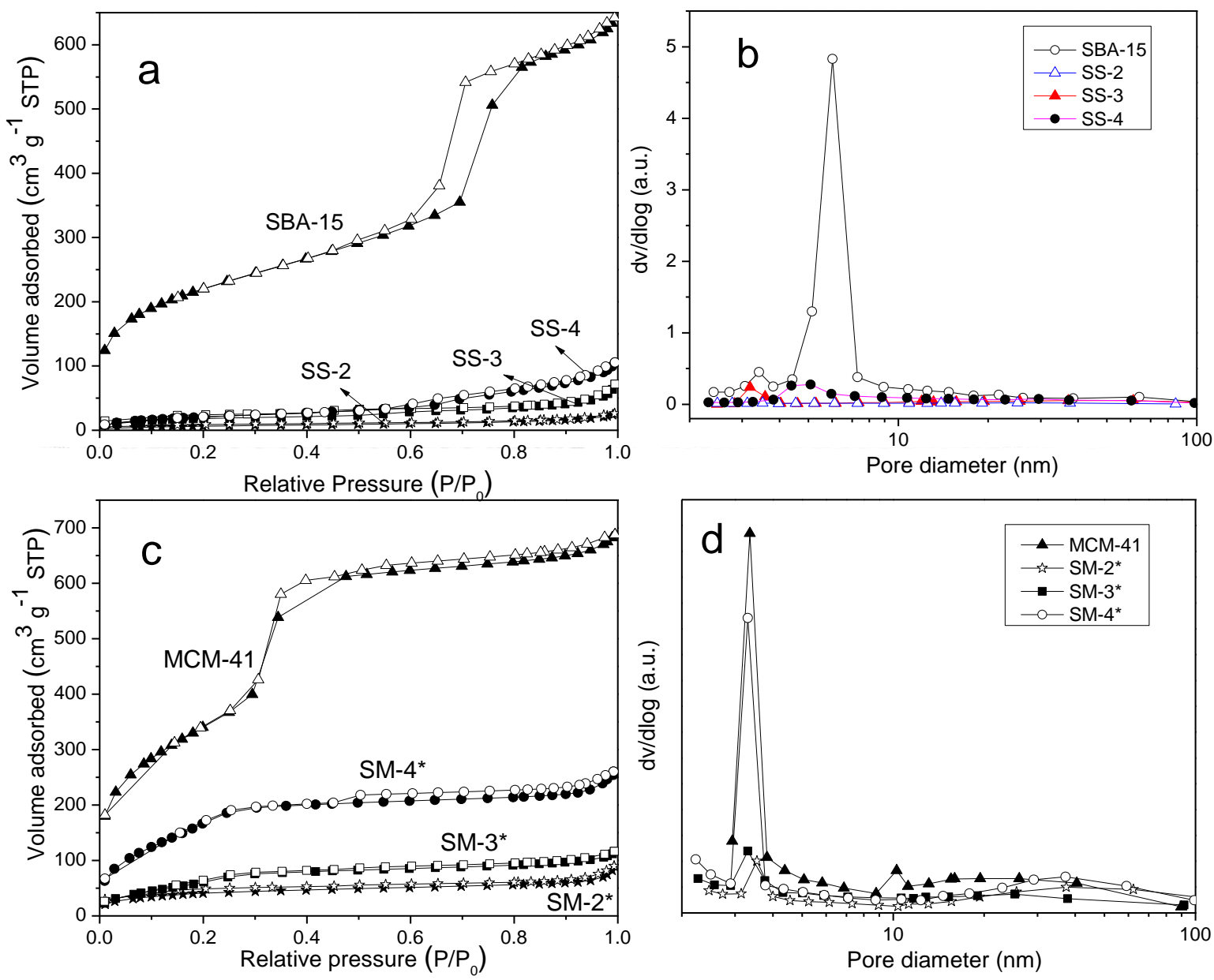

Fig. 2. The $\mathrm{N}_{2}$ adsorption isotherm of (a, b) SBA-15 and (c, d) MCM-41 composites. 


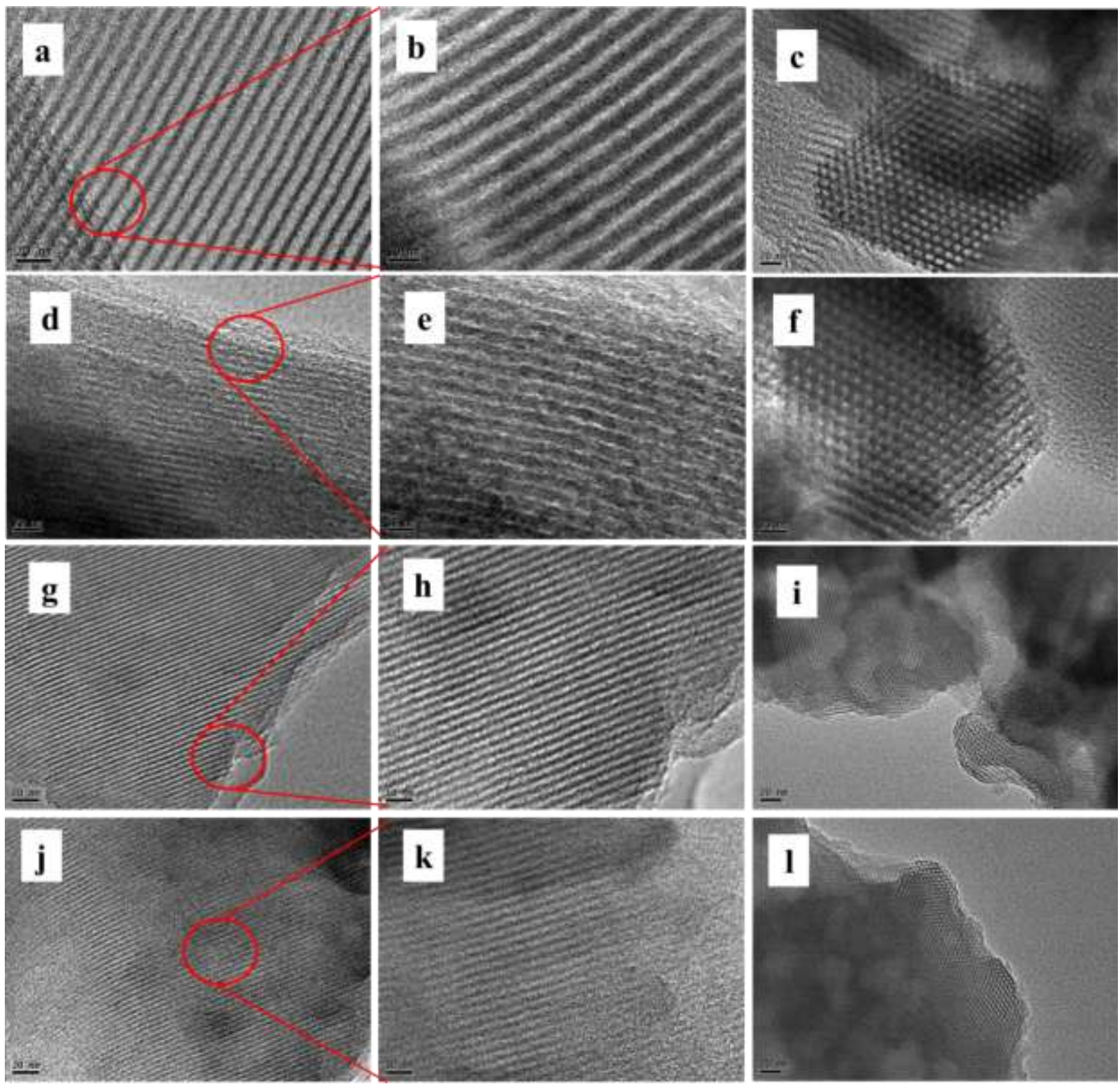

Fig. 3. TEM images of (a, b, c) SBA-15, (d, e, f) SS-3, (g, h, i) MCM-41 and (j, k, l) SM-3 samples. 


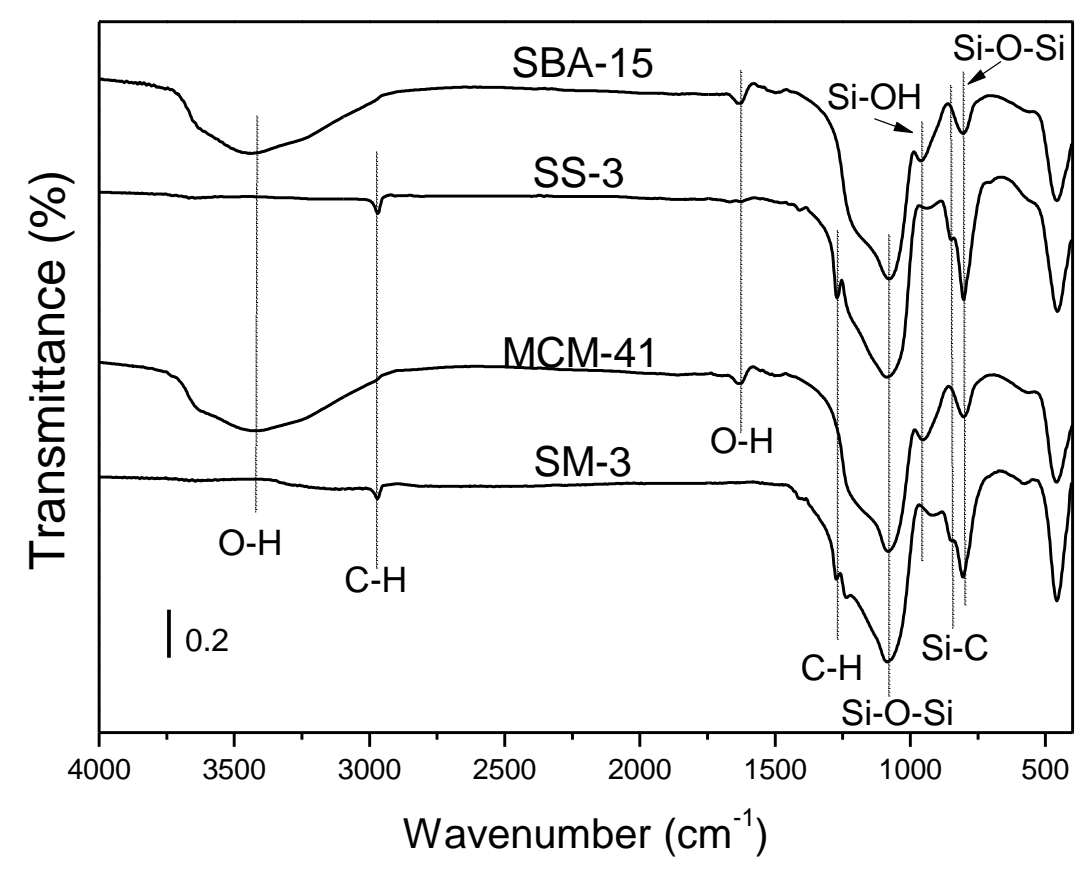

Fig. 4. FT-IR spectra of SBA-15, SS-3, MCM-41 and SM-3 samples.

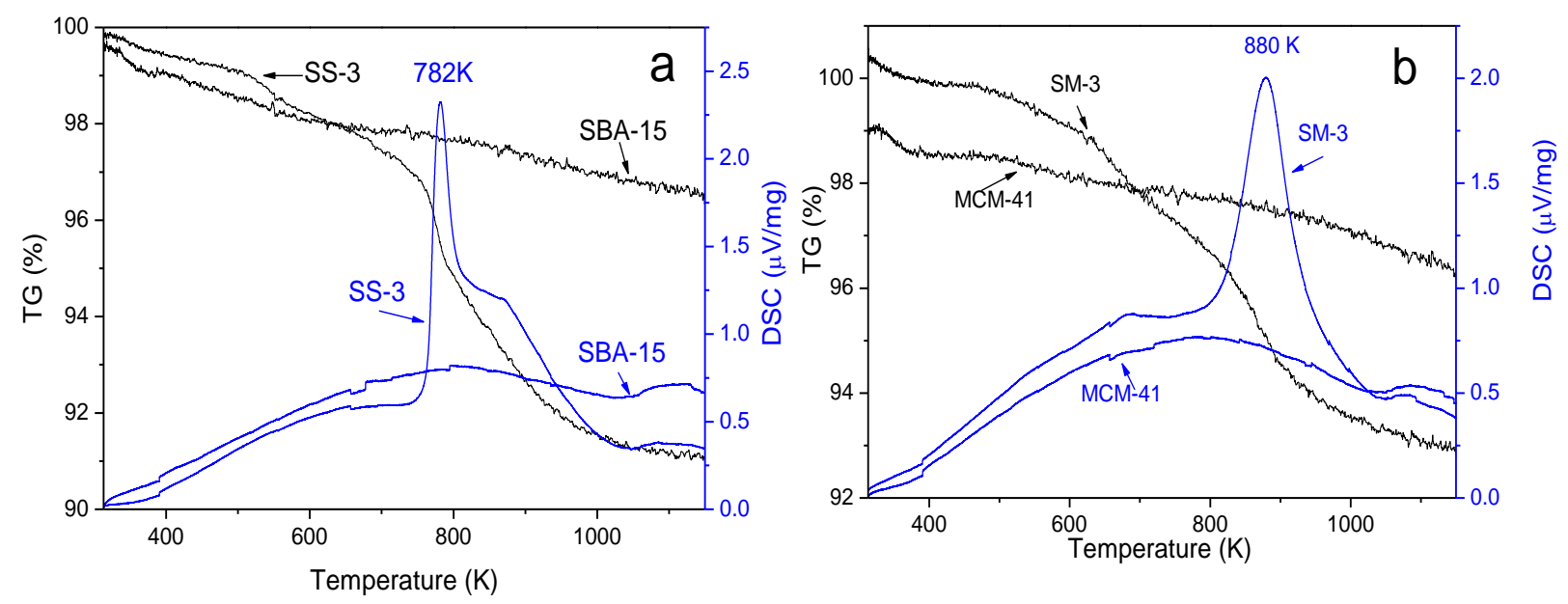

Fig. 5. The TG-DSC plot of (a) SBA-15 and SS-3, (b) MCM- 41 and SM-3 samples. 

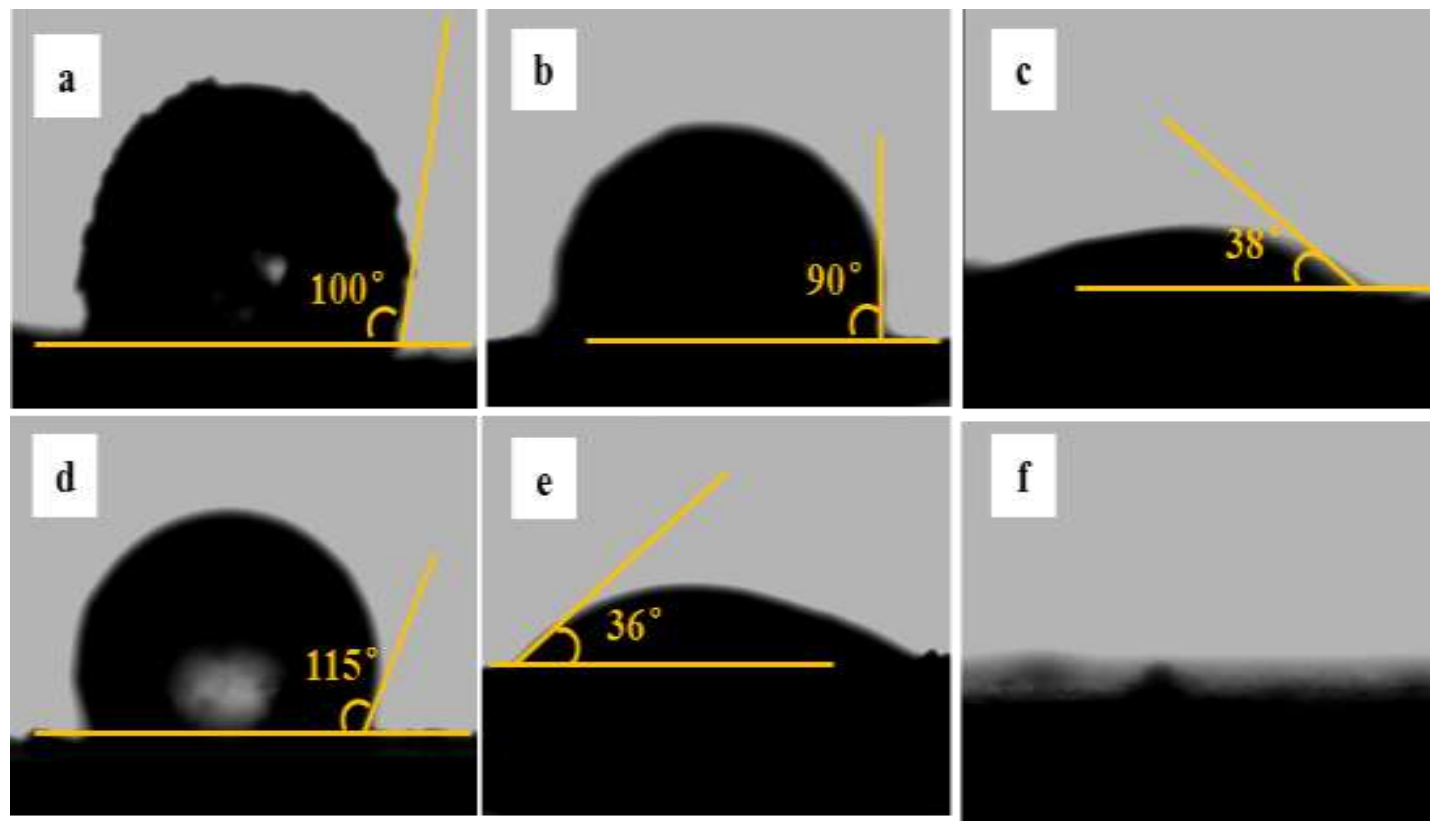

Fig. 6. Contact angle images of (a) SM-2, (b) SM-3, (c) SM-4, (d) SS-2, (e) SS-3 and (f) SS-4 samples.
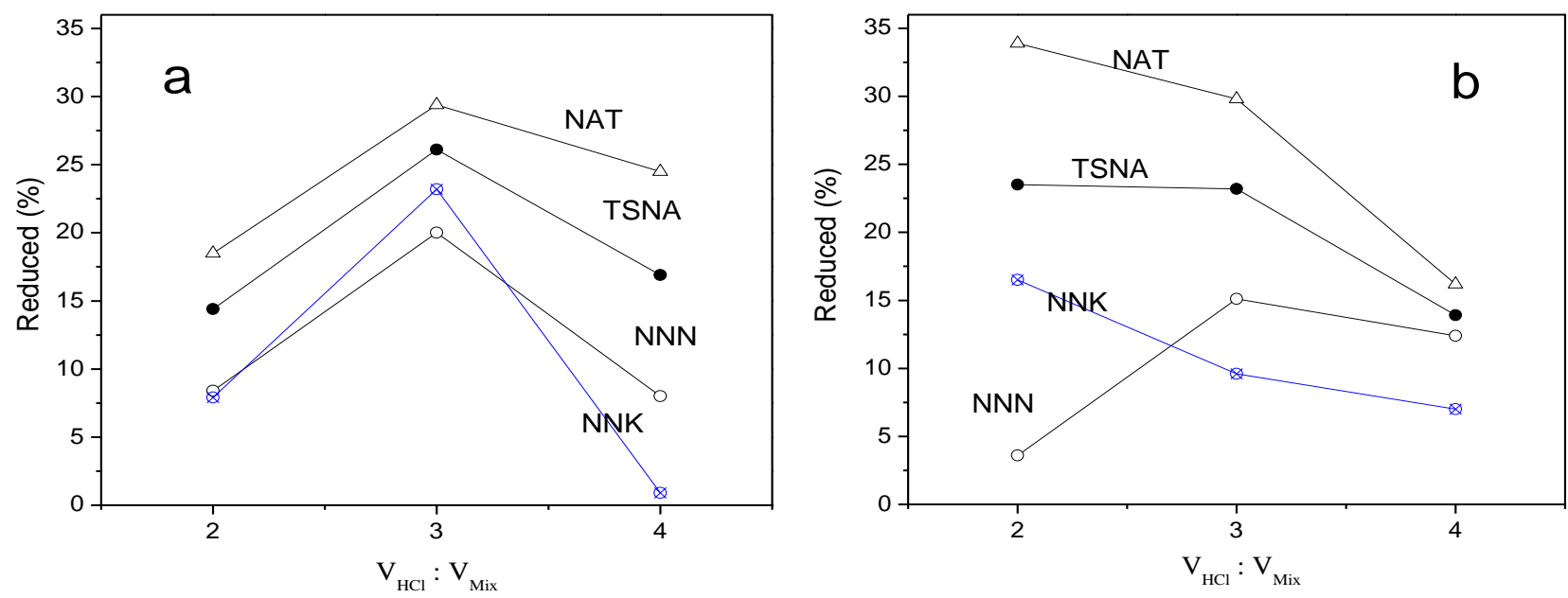

Fig. 7. Adsorption of TSNA by SS-r (a) and SM-r (b) samples in tobacco extract solution. 